\title{
Estrategias operativas de compañías de generación en mercados Spot de electricidad
}

\section{Generation Companies' Operative Strategies in the Spot Electricity Market}

\author{
Liera-Moreno E.V. \\ Departamento de Distribución \\ Comisión Federal de Electricidad \\ Correo:limor237@hotmail.com \\ Gutiérrez-Alcaraz G. \\ Instituto Tecnológico de Morelia, Michoacán \\ Departamento de Ingeniería Eléctrica y Electrónica \\ Correo:ggutiera@gmail.com
}

\author{
Tovar-Hernández J.H. \\ Instituto Tecnológico de Morelia, Michoacán \\ Departamento de Ingeniería Eléctrica y Electrónica \\ Correo:horaciotovar@mexico.com
}

Información del artículo: recibido: abril de 2010, aceptado: junio de 2011

\section{Resumen}

En la regulación tradicional se asumía explícitamente la obligación de satisfacer la demanda del consumidor, lo cual garantizaba a las compañías generadoras la recuperación total de sus costos. Sin embargo, con el objetivo de lograr una mayor eficiencia, reducir el precio de la electricidad, enfrentar el continuo y creciente consumo de electricidad e igualar precios en diferentes regiones, se ha creado una nueva estructura de la industria eléctrica, donde la energía se comercializa a través de un mercado. Es la participación día a día en el mercado la que determina los flujos de caja futuros para una compañía de generación, con los cuales debe enfrentar todos sus compromisos financieros y económicos. En este trabajo se estudian los ingresos de flujos de efectivo futuros requeridos para cumplir con un perfil de acciones esperadas por una compañía de generación. Para este propósito, los flujos de caja futuros se consideran dependientes de un solo activo: la energía eléctrica. Se genera una serie de escenarios con variación en los precios de combustible, a fin de estimar los flujos de caja futuros de una compañía de generación. La respuesta de participación en el mercado por parte de las compañías de generación competidoras se considera en cada escenario. La variación de precios de combustible se modela mediante un árbol binario concurrente.

\section{Descriptores}

- asignación de unidades

- compañías de generación

- mercados de electricidad

- portafolio

- riesgo

- subastas 


\begin{abstract}
In traditional regulation the obligation to meet the consumer demand was assumed, this guaranteed to generation companies the full recovery of their costs. However, in order to achieve greater efficiency, reduce the price of electricity, meet the continuously growing electricity consumption, and equalize prices in different regions, a new structure of the electricity industry has been created, where electric energy is traded through a market. Generation company's future cash flows depend on day to day market participation, in order to satisfy all of their financial and economic requirements. In this paper, future cash flows required to fulfill with economic and financial commitments by a generation company immerse in this new market structure are studied. For this purpose, future cash flows are considered to be dependent on a single asset: electricity. Several scenarios with different fuel prices are generated in order to estimate the generation company's future cash flows. The response of the competing generation companies is taken into account at each scenario. The fuel price changes are modelled using a concurrent binary tree.
\end{abstract}

\section{Introducción}

Durante las últimas décadas se han vivido cambios en la organización de mercados a nivel mundial, la tendencia ha sido privatizar empresas paraestatales $y$, como consecuencia, se ha observado una fuerte tendencia hacia la liberación de los mercados de energía, incluyendo bienes como el gas natural, petróleo, carbón, y la electricidad, así como toda la infraestructura asociada con su transporte.

En países en desarrollo, la privatización y liberación se ha visto como una opción necesaria para sobreponerse a la inercia organizacional que exige mayores incentivos, por un lado, mientras que por el otro, requiere inversiones económicas cuantiosas y cada vez mayores (Joskow, 2005). También, se ha considerado que la liberación es atractiva para la inversión privada, que a la postre contribuye a la rápida satisfacción de una demanda energética continuamente creciente. En algunos otros casos, la liberación se ha visto como un medio que provee competitividad en mercados locales, como el caso de aerolíneas, telecomunicaciones, transporte terrestre, por mencionar algunas (Joskow, 2005).

En el sector eléctrico, con la separación de las actividades de generación con las de transmisión, se posibilitó la competencia en la primera de éstas. Por otra parte, los sistemas de transmisión, teniendo una economía de escala ${ }^{1}$ significativa pueden ser vistos como un monopolio natural pero con un acceso abierto al servicio de transporte electricidad (Bhattacharya et al., 2001).

\footnotetext{
${ }^{1}$ La economía de escala se refiere al poder que tiene una empresa cuando alcanza un nivel óptimo de producción para producir más a menor costo.
}

Por lo general, la restauración de los sectores eléctricos tiene en su estructura básica de diseño al mercado primario de compraventa de energía eléctrica como denominador común. Sin embargo, la organización en la provisión de la transmisión y otros servicios auxiliares difiere, ya que el suministro de éstos se adecua a las necesidades específicas de cada sistema. Existen varias formas de diseñar un mercado de electricidad, por lo que prácticamente no hay dos modelos idénticos; no obstante, estos pueden agruparse en modelos centralizados, descentralizados e híbridos (Wilson, 2001).

En el modelo centralizado, el mercado eléctrico funciona a través de una optimización centralizada y detallada de los recursos de generación, los cuales conforman un pool para satisfacer la demanda de electricidad de la manera más económica posible, considerando una serie de restricciones asociadas a la red eléctrica (Wilson, 2001). Dicha optimización se lleva a cabo a través de una "asignación de unidades de generación", que consiste en determinar cuáles unidades deberán ser sincronizadas y a qué niveles de potencia deben producir, de tal manera que se suministre la demanda al menor costo posible, todo esto dentro de las limitaciones físicas y operativas de la red de transmisión y de las unidades generadoras. Un operador del sistema (SO) ejecuta la asignación de unidades, supervisa la operación del sistema de transmisión y la comercialización de servicios auxiliares. El pool recibe las ofertas de los generadores que contienen una función de costo y un conjunto de restricciones operacionales. La función de costo contiene los coeficientes de costo sin carga, costo de encendido y costo variable, mientras que en las restricciones operativas se incluyen las potencias mínima 
y máxima de cada unidad de generación, restricciones de rampa y tiempo mínimo fuera, entre otras. Una vez resuelto el problema de asignación de unidades, el pool calcula diferentes componentes de precio para determinar los precios para el proveedor y el consumidor; entre esas componentes está el precio marginal del sistema, el cual define el precio para la potencia activa y se calcula de tal forma que las compañías de generación recuperen el total de sus costos. Un modelo centralizado fue utilizado por Inglaterra y Wales Power Pool (EWPP) como el mecanismo del mercado (Wilson, 2001).

Los modelos descentralizados nacen con el objetivo de crear un mecanismo transparente de comercialización de la energía. Lo anterior, debido a las fuertes críticas del modelo centralizado por la complejidad del mecanismo de mercado y el requerimiento de información (Wilson, 2001). En un modelo descentralizado, se separan las actividades del mercado de las actividades de la operación del sistema eléctrico de potencia. En los primeros modelos de mercado de este tipo, como en el caso del mercado de California, se tenían dos operadores: el operador de mercado (MO) y el operador independiente del sistema (ISO). El operador del mercado era el Power Exchange (PX), el cual recibía las ofertas simples (precio y cantidad) que los proveedores y consumidores enviaban un día en adelanto para comprar y vender potencia activa. Para cada hora, el PX ordenaba las ofertas para construir las curvas de suministro/demanda, cuya intersección define la asignación de unidades y el precio Spot de mercado. Una vez realizada la subasta, el PX enviaba las potencias aceptadas al ISO, quién determinaba si eran factibles o no, desde el punto de vista de red de transmisión. La actuación de las compañías de generación en este tipo de mercado necesita basarse en sus estrategias de oferta para recuperar todos sus costos y, al mismo tiempo, tales estrategias tienen que ser diseñadas para que los posibles resultados de la subasta observen las restricciones operativas de sus unidades. Por otra parte, es posible que un solo operador realice ambas tareas como el caso del Independent Electricity Market Operator de la provincia de Ontario (Wilson, 2001).

El modelo centralizado conlleva a menores costos de transición y a una operación confiable del sistema; a pesar de esto, se discute que este modelo no permite una operación transparente del mercado por las sofisticaciones matemáticas que representa la determinación de precios de mercado. En un modelo descentralizado, los mecanismos de subasta son la forma más sencilla, eficiente y transparente de calcular el equilibrio del mercado (precio y cantidad total del mercado) (Wilson, 2001).
Recientemente se han creado modelos híbridos, debido a que los mercados no son perfectamente competitivos. Estos modelos no realizan la asignación de unidades en el mercado primario, pero permiten ciertas condiciones operativas que se expresan en las ofertas, además de que consideran una representación simple de la red de transmisión, de manera que el modelo generalmente se resuelve mediante técnicas de programación lineal. La base de este modelo es la teoría de fijación de precios Spot. El problema ahora consiste en una maximización del beneficio social y su solución provee una asignación de precios y potencias asociadas para proveedores y consumidores en forma simultánea, donde la variable de optimización dual asociada a las restricciones de demanda determina el precio del mercado (Wilson, 2001).

La incertidumbre y riesgos inherentes en los mercados eléctricos liberalizados son mucho más visibles que en un modelo verticalmente integrado. Dentro de los riesgos e incertidumbre a los que se exponen los participantes en un mercado primario de energía se incluyen:

- Precio de la electricidad en el mercado primario.

- Precio de mercado de la energía primaria (carbón, gas y petróleo).

- Tecnología (grandes centrales térmicas o plantas de ciclo combinado, generación distribuida).

- Comportamiento de los competidores.

- Disponibilidad de plantas.

- Crecimiento de la demanda.

- Regulación y contexto político.

Se han propuesto varios métodos para la estimación de las ofertas de compañías de generación, desde la teoría de portafolios hasta la teoría de juegos. Sin embargo, el problema de asignación de unidades sigue siendo fundamental para el desarrollo de las estrategias de mercado de las compañías de generación con sus respectivas adecuaciones (Hobbs et al., 2001). El problema de asignación de unidades ha sido tema de investigaciones por décadas. A lo largo de los años, la industria eléctrica ha utilizado diferentes técnicas de optimización para dar solución al problema, las cuales abarcan desde lista de prioridades, programación dinámica, relajación Lagrangiana (Wollenberg y Ching, 1996) hasta modelos meta heurísticos (Paiva, 1999), algoritmos genéticos (Gwo, 2004), sistemas expertos y redes neuronales (Dimitris, 2006). Una extensa lista de literatura puede encontrarse en (Padhy, 2004) y (Gerald et al., 1994).

En un ambiente de competencia las compañías de generación no sólo considerarán las restricciones 
operativas propias del generador, sino que toman en cuenta las restricciones del sistema de transmisión (capacidad, precios nodales, etcétera) (Hossein et al., 2008) y de servicios auxiliares (reserva de potencia activa, control de voltaje, etcétera) (Zuyi, 2005). Sin embargo, en la actualidad no existe un mercado competitivo sino un mercado de competencia oligopólica. En Gutierrez (2008), se presenta la decisión de producción de compañías de generación en un entorno de oligopolio, el modelo está basado en un equilibrio estático, el cual se resuelve secuencialmente en el tiempo. Para cada paso de tiempo, el problema se formula y resuelve independientemente usando el modelo de Cournot, además de agregar restricciones de encendido/apagado y rampas arriba/abajo. Debido a la competencia con otras compañías de generación se debe tener un buen pronóstico de oferta/demanda para que los generadores de una compañía queden dentro de las unidades despachables. En Wen (2001), se modela el problema óptimo de estrategias de oferta para proveedores competitivos en un mercado de 24 horas en adelanto. Ya que la oferta/ demanda determina el equilibrio del mercado, precio y cantidad, es importante estimar el precio de mercado. El precio de electricidad es un punto de referencia en la industria eléctrica, el cual se ve afectado por los cambios en los costos de combustible. Chung (2000) usa parte de la selección de una estructura real para evaluar la flexibilidad de operación de las unidades de potencia, dicha operación se formula como un problema estocástico de escenarios múltiples suponiendo un mercado Spot, tanto para el precio de combustible como para el de electricidad, generando un árbol de precios. Para el modelado del árbol de precios se utiliza programación dinámica estocástica. En consecuencia a la variación de los precios, se tienen riesgos que afectan las ganancias de una compañía de generación, por lo que se debe incluir la incertidumbre en los precios (entre otros aspectos) como se analiza en Hossein et al. (2008). Para controlar este tipo de riesgo o diversificarlo, se ha propuesto la aplicación de herramientas financieras. Fusaro (1998), presenta algunas herramientas que permiten la medición del riesgo (VaR, Value at Risk), la aplicación de herramientas financieras que diversifican el riesgo y una descripción de cómo se han utilizado en el sector eléctrico. Blaesing (2007) propuso el uso de herramientas estocásticas para la optimización del problema de asignación de unidades y de decisiones de mercado. El método de evaluación consiste en la descomposición del horizonte de valoración de las unidades de generación dentro de subperiodos, donde los costos e ingresos se cuantifican sucesivamente. Además, considera estrategias de gestión de riesgo que optimizan la asignación de unidades y decisiones de mercado para diferentes preferencias de riesgo.

La necesidad de tener una mayor eficiencia económica para las compañías de generación en el sector eléctrico, bajo un ambiente de competencia e incertidumbre, las obliga a desarrollar nuevas herramientas que permitan determinar de manera óptima su forma de operar. En este trabajo se desarrolla un modelo estocástico para el manejo de la incertidumbre en los precios de combustible y electricidad con el objetivo de determinar la toma de decisión en la participación de la producción de las compañías de generación.

El resto del documento está organizado como se describe a continuación. En la siguiente sección se presenta una descripción del mercado spot de electricidad y en la sección que le sigue la formulación matemática del problema. La descripción de la metodología de simulación se muestra después y, a fin de ilustrar la aplicación de esta metodología, se integra un ejemplo numérico. Finalmente, se resumen las conclusiones asociadas a los resultados obtenidos.

\section{Mercado Spot de electricidad}

En mercados eléctricos descentralizados, los mecanismos de subasta son la forma más sencilla, eficiente y transparente de calcular el equilibrio del mercado (Sheblé, 1999). Un mecanismo de subasta está constituido por un conjunto de reglas definido, que determina el equilibrio del mercado con base en ofertas proporcionadas por los diferentes participantes: productores y consumidores.

El mercado Spot de electricidad consiste en una casa de subastas, representada por el operador del mercado y un determinado número de oferentes, que representan a las compañías de generación (GenCo). Estas compañías de generación realizan sus propias decisiones de participación en el mercado, basadas en la maximización de ganancias esperadas, dada la información de que disponen. Las compañías de generación ofertan su producción siempre y cuando el precio del mercado sea mayor a sus costos marginales de producción. La subasta se realiza de la siguiente manera: el subastador (operador del mercado) anuncia una demanda a satisfacer. Cada GenCo presenta una oferta, que es un par ordenado $(p, q)$, donde $p$ es el precio al cual está dispuesto a vender su producción y $q$ la cantidad ofertada. El operador del mercado clasifica las ofertas en orden ascendente por precio, agregando la cantidad de cada oferta a un total en ejecución hasta que este total satisface la demanda, la cual, en este caso, no es ofertada por consumidores. En este momento se tiene el equilibrio del 
mercado y de la última oferta de generación se acepta sólo la cantidad que iguala a la demanda. El proceso de subasta se muestra en la figura 1.

Una vez que se determina el equilibrio del mercado, de precio y cantidad de mercado, las ofertas están comprometidas y los contratos se establecen. Ahora, cualquier GenCo cuya oferta fue aceptada está obligada a proporcionar la cantidad de electricidad aceptada.

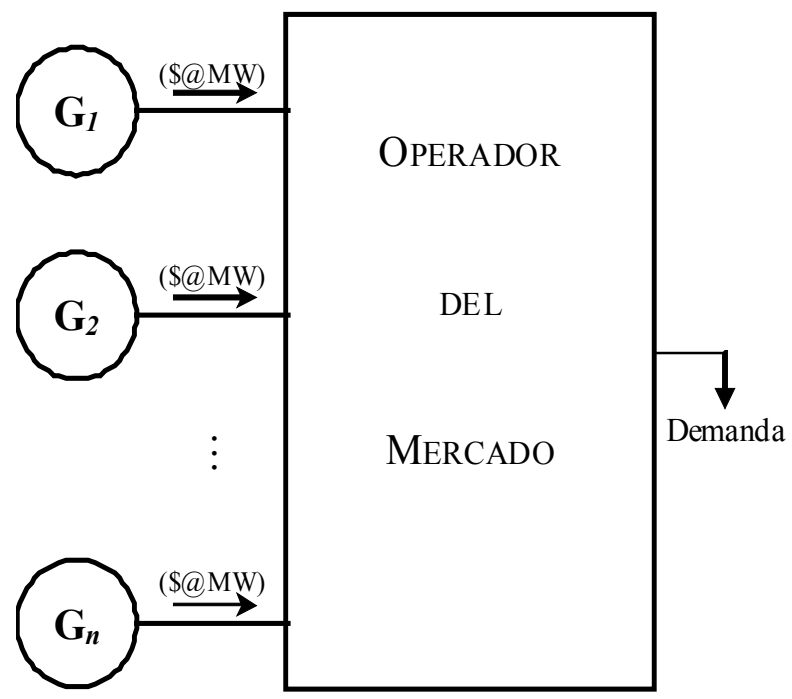

Figura 1. Subasta en un mercado Spot de electricidad
El mecanismo de subasta de un solo lado, descrito antes, se ha utilizado para la determinación del equilibrio de mercado donde solamente un tipo de participante oferta, generalmente productores (Vijay, 2002). Sin embargo, existen también las subastas de doble lado, en donde tanto productores como consumidores ofertan (Vijay, 2002).

A manera de ilustración, considere que 9 compañías de generación participan en el mercado Spot de electricidad. Las GenCos envían sus ofertas mostradas en la tabla 1 al operador del mercado. La demanda del sistema eléctrico a satisfacer, conocida por el operador del mercado, es $135 \mathrm{MW}$.

Tabla 1. Ofertas realizadas por las GenCos participantes

\begin{tabular}{lccccccccc}
\hline GenCo & A & B & C & D & E & F & G & H & I \\
\hline Cantidad $(M W)$ & 20 & 30 & 25 & 20 & 10 & 35 & 20 & 15 & 30 \\
Precio $(\$ / M W h)$ & 5 & 20 & 8 & 10 & 22 & 25 & 45 & 48 & 40
\end{tabular}

En la tabla 1 se observa que las ofertas de las GenCo varían en precio y cantidad, ya que dependen de las estrategias de cada GenCo. La oferta de menor precio corresponde a la GenCo A, mientras que la de mayor precio proviene de la GenCo H. El operador del mercado apila las ofertas en función del precio, como se muestra en la figura 2, donde se observa que la cantidad aceptada por el mercado es 30 de los 35 MW que ofertó la GenCo F.

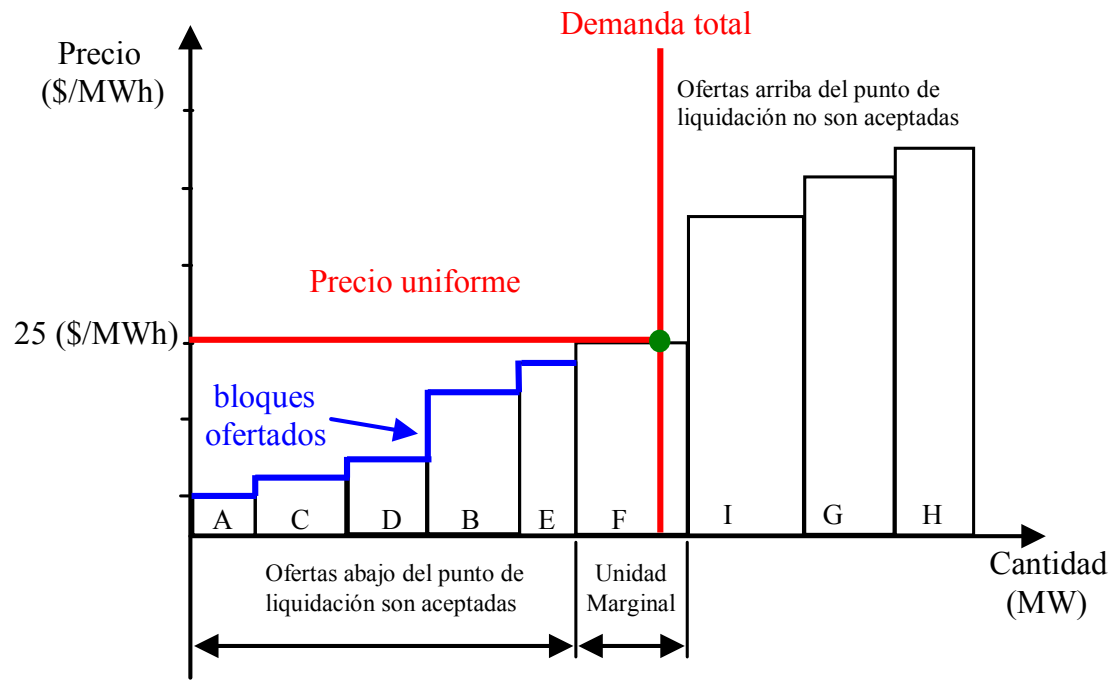

Figura 2. Subasta de un solo lado 
Existen dos formas de establecer el precio del mercado de electricidad para las retribuciones de las compañías de generación. Una forma es fijar el precio a la oferta de cada GenCo, el cual se conoce con el nombre de pago al precio ofertado (Pay as Bid). En este modelo, los oferentes que ganan, reciben como precio del mercado su valor ofertado. Es decir, existe una diferencia de precios en el mercado. Por ejemplo, en la figura 2 se observa que la oferta aceptada de la GenCo A será retribuida a un precio menor que las ofertas aceptadas del resto de las GenCos. Para el caso de la oferta aceptada de la GenCo D, ésta es retribuida a un precio mayor, con respecto a las Gen Cos A y C, pero menor que las GenCos B, E y F.

Otra forma, es el Precio Uniforme (PU). El Precio Uniforme es el valor de la última oferta que cubre la demanda del mercado, es decir, existe un precio único de mercado. En una subasta de PU, todos los oferentes ganadores son retribuidos a precio uniforme, independientemente del valor del precio ofertado (Zou et al., 2004).

Continuando con el ejemplo, los ingresos de las Gen $\operatorname{Cos}$ bajo un mecanismo de precio discriminatorio se reportan en la tabla 2.

Tabla 2. Ingresos de las GenCos a precio discriminatorio

\begin{tabular}{lccccccccc}
\hline GenCo & A & B & C & D & E & F & G & H & I \\
\hline Cantidad (MW) & 20 & 30 & 25 & 20 & 10 & 30 & 0 & 0 & 0 \\
Ingresos (\$) & 100 & 600 & 200 & 200 & 220 & 750 & 0 & 0 & 0 \\
\hline
\end{tabular}

En la tabla 2 se observa que las GenCos G, H e I tienen ingresos nulos, dado que sus ofertas no fueron aceptadas por el mercado. La GenCo F es la que tiene mayores ingresos, mientras que la que obtiene menores ingresos resulta ser la GenCo A.

Los ingresos de las GenCos bajo un esquema de precio uniforme se reportan en la tabla 3.

Tabla 3. Ingresos de las GenCos a precio uniforme

\begin{tabular}{lccccccccc}
\hline GenCo & A & B & C & D & E & F & G & H & I \\
\hline Cantidad (MW) & 20 & 30 & 25 & 20 & 10 & 30 & 0 & 0 & 0 \\
Ingresos (\$) & 500 & 750 & 625 & 500 & 250 & 750 & 0 & 0 & 0 \\
\hline
\end{tabular}

En la tabla 3 se aprecia que, al igual que en el caso anterior, las Gen Cos con ingresos nulos son G, H e I, ya que sus ofertas quedaron por arriba del equilibrio del mercado. La GenCo E presenta menores ingresos, mientras que las GenCos B y F son las que obtienen los mayores ingresos.

\section{Formulación del problema}

La estimación de las ofertas de una compañía de generación está sujeta a restricciones operativas y temporales de cada una de las unidades que conforman la planta de generación, por lo que el problema de asignación de unidades sigue siendo la herramienta básica para la toma de decisiones de producción de compañías de generación.

Desde un punto de vista de operador del sistema (modelo centralizado), el problema de asignación de unidades se formula como:

$$
\begin{aligned}
& \operatorname{Minimizar} \sum_{t=1}^{T} \sum_{i=1}^{n}\left\{C_{i}\left(P g_{i, t}\right)+S u_{i}+S d_{i}\right\} u_{i, t} \\
& \sum_{i=1}^{n} P g_{i, t} u_{i, t}=D_{t} \quad \forall t=1, \ldots, T \\
& \sum_{i=1}^{n} S_{i, t} \leq S_{R, t} \quad \forall t=1, \ldots, T \\
& \begin{array}{l}
P g_{i, t+1}-P g_{i, t} \leq Z_{i} \quad \forall t=1, \ldots, T \\
P g_{i, t}-P g_{i, t+1} \leq W_{i}
\end{array} \\
& P g_{i}^{\min } \leq P g_{i} \leq P g_{i}^{\max } \\
& \left(x_{i, t-1}^{o n}-t_{i}^{o n}\right)\left(u_{i, t-1}-u_{i, t}\right) \geq 0 \\
& \left(x_{i, t-1}^{\text {off }}-t_{i}^{\text {off }}\right)\left(u_{i, t-1}-u_{i, t}\right) \geq 0
\end{aligned}
$$

donde:

$C_{i}\left(P g_{i, t}\right)$ es el costo de producción del generador $i$ en el periodo $t$,

$P g_{i, t} \quad$ es la potencia generada por la unidad $i$ en el periodo $t$;

$D_{t} \quad$ es la demanda en el periodo $t ;$

$S_{i, t} \quad$ es la reserva rodante del generador $i$ en el periodo $t$,

$P g_{i, t}{ }^{\max }$ y $P g_{i, t}{ }^{\min }$ son los límites operativos, máximo y mínimo de la unidad $i$ en el periodo $t$,

$n \quad$ es el número total de unidades de la compañía de generación,

$S_{R, t} \quad$ es la reserva total de generación de potencia activa del sistema en el periodo $t$,

$S u_{i} \quad$ son los costos de arranque de la unidad $i$,

$S d_{i} \quad$ son los costos de apagado de la unidad $i$,

$Z_{i} \quad$ es la rampa arriba de la unidad $i$,

$W_{i} \quad$ es la rampa debajo de la unidad $i$,

$t_{i}^{\text {on }}, t_{i}^{\text {off }}$ son los tiempos mínimos, en horas, que la unidad debe estar prendida/apagada, 
$u_{i, t}$

es una variable binaria que indica la condición de operación de unidad $i\left(u_{i, t}=0\right.$ indica que la unidad está apagada y $u_{i, t}=1$ que la unidad está encendida) en el periodo $t$.

La reestructuración ha modificado el problema de asignación de unidades dado que en este nuevo ambiente se tienen varias compañías de generación, por lo que cada compañía es responsable de sus decisiones de producción y, por ende, de su participación en el mercado, es decir, las compañías de generación eligen tanto el nivel de producción como la hora de entrega al sistema. Así, la redefinición del problema de asignación de unidades en un ambiente competitivo involucra cambios en la función objetivo y en las restricciones de demanda. Las restricciones operativas y temporales deberán seguir satisfaciéndose. Por lo anterior, el nuevo problema de asignación de unidades es:

$$
\begin{array}{lc}
\text { Maximizar } \sum_{t=1}^{T} \sum_{i=1}^{n}\left\{P g_{i, t} p_{E t}-C_{i}\left(P g_{i, t}\right)-S u-S d\right\} u_{i, t} \\
\sum_{i=1}^{n} P g_{i, t} u_{i t} \leq D_{t} & \forall t=1, \ldots, T \\
\sum_{i=1}^{n} S_{i, t} \leq S_{R} & \forall t=1, \ldots, T \\
P g_{i, t+1}-P g_{i, t} \leq Z_{i} & \forall t=1, \ldots, T
\end{array}
$$

$$
\begin{aligned}
& P g_{i, t}-P g_{i, t+1} \leq W_{i} \quad \forall t=1, \ldots, T \\
& P g_{i, t}^{\min } \leq P g_{i, t} \leq P g_{i, t}^{\max }
\end{aligned}
$$

$$
\begin{aligned}
& \left(x_{i, t-1}^{o n}-t_{i}^{o n}\right)\left(u_{i, t-1}-u_{i, t}\right) \geq 0 \\
& \left(x_{i, t-1}^{o f f}-t_{i}^{o f f}\right)\left(u_{i, t-1}-u_{i, t}\right) \geq 0
\end{aligned}
$$

Como ahora las compañías de generación eligen el nivel de producción y la hora de entrega, éstas tienen que elegir el nivel de producción que les permita recuperar sus gastos, como: compra de combustible, pago de trabajadores, mantenimiento a las unidades o gastos que se llegan a tener por alguna dependencia financiera, entre otros, los cuales no necesariamente se introducen en la función de costo. La figura 3 muestra un diagrama del flujo de caja esperado para una compañía de generación.

Los mercados de electricidad son en realidad mercados de competencia oligopólica, en donde las decisiones de los agentes se realizan en forma secuencial y repetitiva. El análisis del comportamiento de los participantes es necesario para el diseño de estrategias de mercado por parte de los productores.

En ciertas circunstancias las GenCos saben poco acerca de las acciones pasadas, de la función objetivo y de las estrategias de competencia de sus oponentes. Cada GenCo puede estimar las decisiones tomadas con anterioridad por sus oponentes, resultado de observar el precio del mercado, ubicación y tipo de combustible

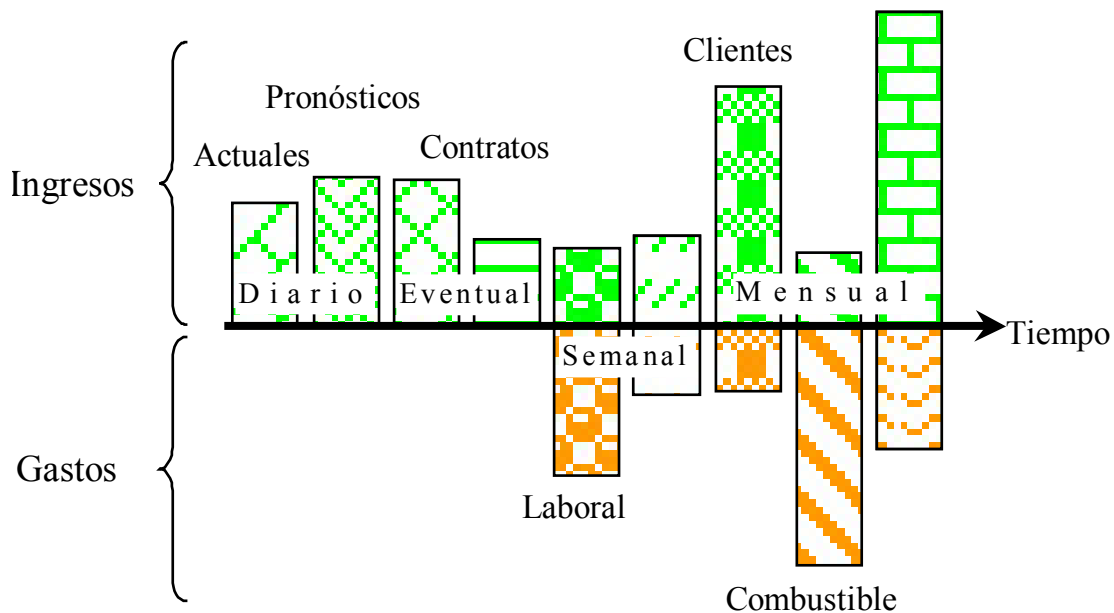

Figura 3. Flujo de caja de una GenCo 


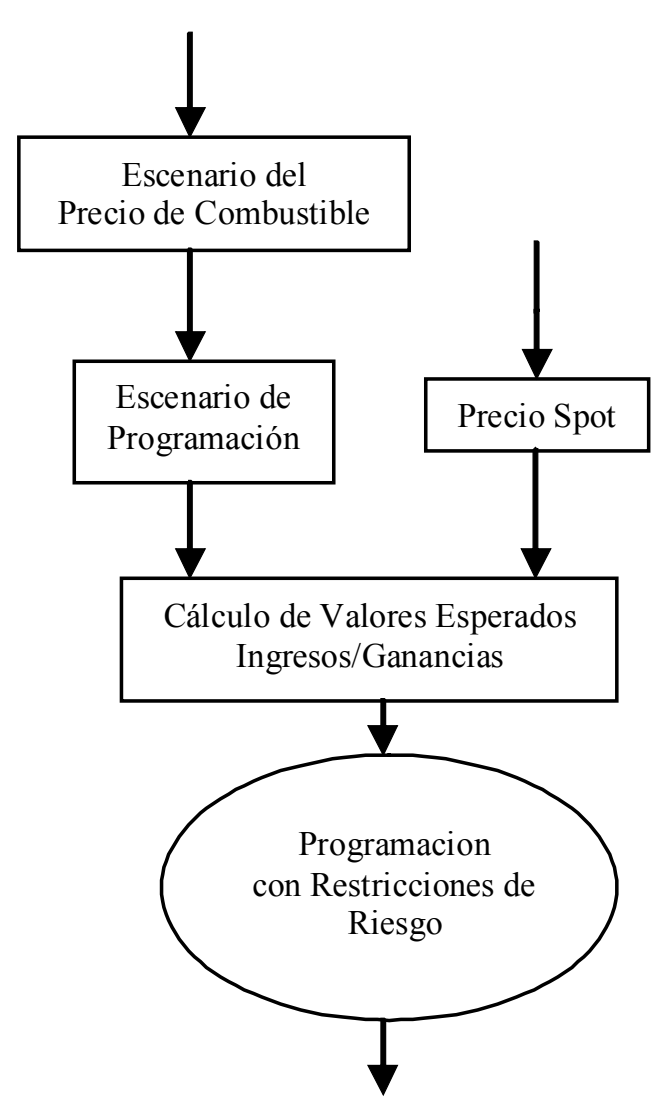

Figura 4. Metodología de solución

de sus rivales. Las GenCos forman un historial del cual acumulan experiencia y les permite la toma de mejores decisiones, decisiones informadas, de producción en periodos futuros con el objeto de maximizar sus ganancias en el corto y largo plazo para poder enfrentar sus compromisos económicos y financieros.

\section{Descripción de la metodología}

Para el desarrollo de las simulaciones realizadas y reportadas, se generó una serie de escenarios con variación en los precios de combustible. Esta variación se modela mediante un árbol binario concurrente (Chung, 2000). Una vez generados los escenarios de precios de combustible, se actualizan las curvas de costo de cada unidad de generación para introducirlas al programa de asignación de unidades. El modelo de asignación de unidades se formula como un problema de optimización entera mixto no lineal y se resuelve mediante un programa desarrollado en GAMS (GAMS, 2004).
Como resultado del problema de asignación se tienen las potencias de generación para cada periodo bajo el escenario en consideración. Adicionalmente, se obtiene la variable dual asociada a la restricción de demanda a suministrar en cada periodo, la cual se compara con el precio Spot de electricidad. Cuando la diferencia del precio Spot con la variable dual es positiva la compañía de generación estará ofertando energía al mercado. Por el contrario, si la diferencia es negativa, la GenCo no ofertará energía al mercado, es decir, las compañías de generación ofertan para vender su producción siempre y cuando el precio del mercado sea mayor a sus costos de producción.

Este proceso se repite para cada escenario. Una vez que se tienen los escenarios evaluados, los resultados se pueden observar mediante un árbol. Aquellos escenarios en los cuales las ganancias esperadas son negativas deberán evitarse en la medida de lo posible.

La metodología se representa en forma esquemática en la figura 4.

Las variaciones en los precios de combustibles y el precio de electricidad se modelan mediante árboles binarios. Se parte de un nodo inicial (día $\mathrm{T}_{\mathrm{o}}$ ), y 2 nodos sucesores en el periodo 1 (día T); cada nodo en el periodo 1 tiene 2 nodos subsecuentes en el periodo 2, y así sucesivamente, tal como se ilustra en la figura 5. El precio de combustible que se obtiene en cada escenario es el mismo para los 24 periodos del día.

De una manera similar, se modelan los precios de electricidad para los casos en los que presentan variaciones.

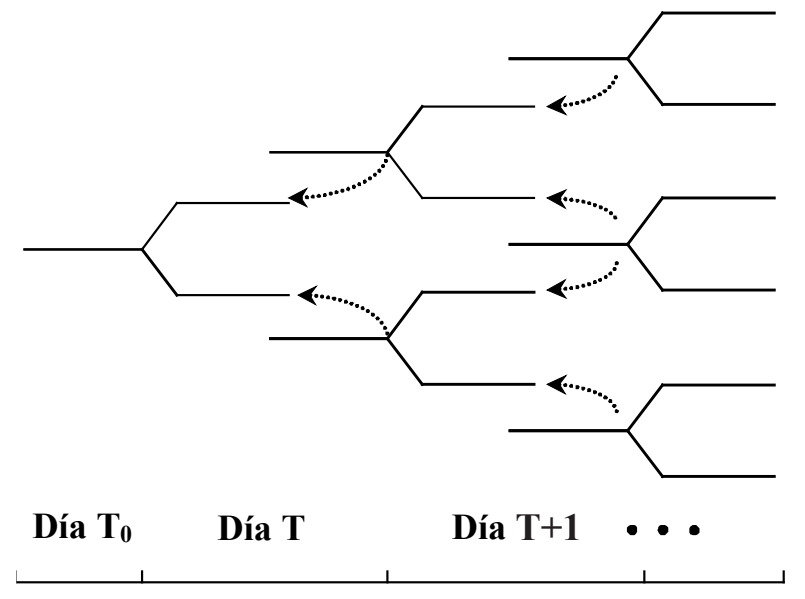

Figura 5. Árbol de escenarios del precio de combustible 
Tabla 4. Especificaciones técnicas de las unidades

\begin{tabular}{|c|c|c|c|c|c|c|c|c|c|c|}
\hline Unidad & $\begin{array}{l}P g^{\min } \\
M W\end{array}$ & $\begin{array}{l}P g^{\max } \\
\mathrm{MW}\end{array}$ & MW/h & $\mathrm{MW} / \mathrm{h}$ & MBtu/h & MBtu/h & MBtu/h & $\begin{array}{c}S u \\
\$\end{array}$ & $\begin{array}{l}t^{o n} \\
\mathrm{~h}\end{array}$ & $\begin{array}{l}t^{\text {off }} \\
\mathrm{h}\end{array}$ \\
\hline 1 & 50 & 200 & 300 & 300 & 29 & 14.8015 & 0.00007 & 67.32 & 15 & 15 \\
\hline 2 & 75 & 330 & 400 & 400 & 72 & 13.5354 & 0.00008 & 95.97 & 20 & 20 \\
\hline 3 & 120 & 370 & 400 & 400 & 49 & 16.2643 & 0.00009 & 101.94 & 18 & 18 \\
\hline 4 & 125 & 495 & 600 & 600 & 82 & 17.2136 & 0.00004 & 114.05 & 15 & 15 \\
\hline 5 & 250 & 570 & 600 & 600 & 105 & 11.1954 & 0.00003 & 134.08 & 16 & 16 \\
\hline 6 & 100 & 600 & 600 & 600 & 100 & 12.1285 & 0.00005 & 141.58 & 18 & 18 \\
\hline
\end{tabular}

\section{Caso de estudio}

En esta sección se presenta un ejemplo de aplicación en donde se utiliza un sistema de 6 unidades, todas pertenecientes a la misma compañía de generación. La tabla 4 muestra parámetros de las curvas de entrada-salida, límites operativos y tiempos mínimos/máximos de encendido/apagado de cada unidad.

La demanda residual que espera ofertar la compañía de generación para los 24 periodos del día en adelanto se muestra en la tabla 5. Los valores de demanda se consideran constantes para los distintos casos.

Dado que los precios de combustible y electricidad varían durante el día, se pueden presentar cuatro escenarios posibles:

a. Incremento en el precio de combustible y de electricidad.

b. Incremento en el precio de combustible y decremento en el precio de electricidad.

Tabla 5. Demanda del sistema

\begin{tabular}{cccc}
\hline Periodo & $\begin{array}{c}\text { Demanda } \\
\text { MW }\end{array}$ & Periodo & $\begin{array}{c}\text { Demanda } \\
\text { MW }\end{array}$ \\
\hline 1 & 1800 & 13 & 1080 \\
2 & 1782 & 14 & 1044 \\
3 & 1746 & 15 & 1026 \\
4 & 1710 & 16 & 1044 \\
5 & 1656 & 17 & 1134 \\
6 & 1683 & 18 & 1242 \\
7 & 1638 & 19 & 1404 \\
8 & 1530 & 20 & 1530 \\
9 & 1359 & 21 & 1638 \\
10 & 1269 & 22 & 1710 \\
11 & 1188 & 23 & 1755 \\
12 & 1134 & 24 & 1791 \\
\hline
\end{tabular}

c. Decremento en el precio de combustible e incremento en el precio de electricidad y

d. Decremento en el precio de combustible y de electricidad.

Gráficamente, estos escenarios se muestran en la figura 6.

Para la simulación de estos cuatro escenarios se aplica diferente tipo de combustible a las unidades, a fin de que el precio sea diferente. Se supone que las unidades 1 y 3 emplean gas, las unidades 2 y 4 carbón y las unidades 5 y 6 combustóleo.

En los cuatro escenarios (A, B, C y D) se considera que el incremento/decremento en cada periodo en el precio del carbón es 5\%, gas y combustóleo es 3\% y el de electricidad es $3 \%$. Se parte de un caso base donde el precio de combustibles es unitario.

Los escenarios de precios en los combustibles muestran una correlación positiva entre ellos; sin embargo, es el precio del carbón el que presenta un mayor incre-

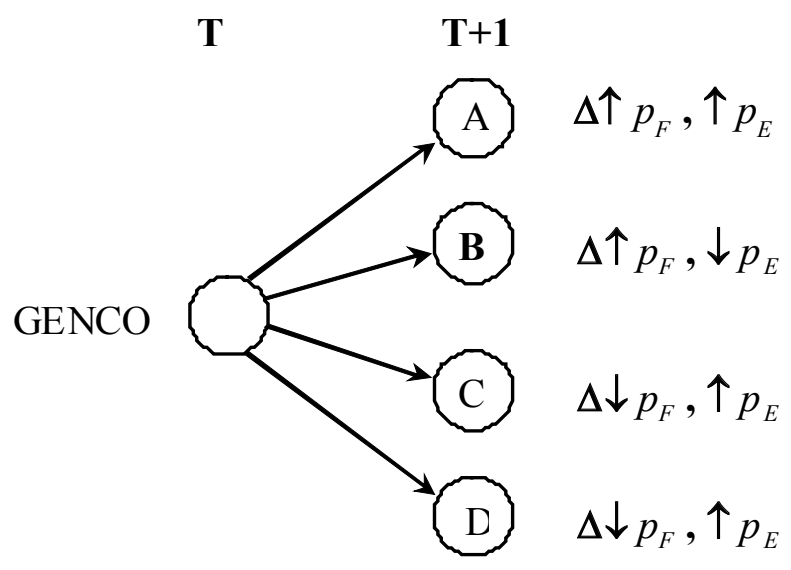

Figura 6. Variación en los precios de combustible y electricidad 


\begin{tabular}{|c|c|c|c|c|c|}
\hline Periodo & $\begin{array}{l}\text { Potencia } \\
\text { (MW) }\end{array}$ & $\begin{array}{c}\text { Precio } \\
\text { (\$/MWh) }\end{array}$ & $\begin{array}{c}\text { Ingresos } \\
(\$)\end{array}$ & $\begin{array}{c}\text { Costos } \\
(\$)\end{array}$ & $\begin{array}{c}\text { Ganancias } \\
(\$)\end{array}$ \\
\hline 1 & 330 & 22.25 & 7340.9 & 4643.36 & 2697.49 \\
\hline 2 & 330 & 22.23 & 7335.9 & 4547.39 & 2788.51 \\
\hline 3 & 330 & 22.23 & 7335.9 & 4547.39 & 2788.51 \\
\hline 4 & 330 & 22.22 & 7331.0 & 4547.39 & 2783.56 \\
\hline 5 & 330 & 22.23 & 7335.9 & 4547.39 & 2788.51 \\
\hline 6 & 330 & 22.25 & 7340.9 & 4547.39 & 2793.46 \\
\hline 7 & 330 & 22.23 & 7335.9 & 4547.39 & 2788.51 \\
\hline 8 & 310 & 20.39 & 6319.4 & 4275.66 & 2043.69 \\
\hline 9 & 189 & 20.36 & 3847.1 & 2633.05 & 1214.045 \\
\hline 10 & 99 & 20.33 & 2012.2 & 1412.79 & 599.385 \\
\hline 11 & 75 & 18.27 & 1370.3 & 1087.61 & 282.64 \\
\hline 12 & 0 & 12.08 & 0.0 & 72 & -72 \\
\hline 13 & 0 & 12.78 & 0.0 & 72 & -72 \\
\hline 14 & 0 & 12.24 & 0.0 & 72 & -72 \\
\hline 15 & 0 & 12.50 & 0.0 & 72 & -72 \\
\hline 16 & 0 & 12.62 & 0.0 & 72 & -72 \\
\hline 17 & 0 & 11.97 & 0.0 & 72 & -72 \\
\hline 18 & 75 & 18.29 & 1371.4 & 1183.58 & 187.795 \\
\hline 19 & 234 & 20.36 & 4763.1 & 3243.66 & 1519.41 \\
\hline 20 & 310 & 20.39 & 6319.4 & 4275.66 & 2043.69 \\
\hline 21 & 330 & 22.23 & 7335.9 & 4547.39 & 2788.51 \\
\hline 22 & 330 & 22.22 & 7331.0 & 4547.39 & 2783.56 \\
\hline 23 & 330 & 22.23 & 7335.9 & 4547.39 & 2788.51 \\
\hline 24 & 330 & 22.25 & 7340.9 & 4547.39 & 2793.46 \\
\hline
\end{tabular}

Tabla 6. Ingresos, costos y ganancias esperadas de la unidad 2 mento con respecto al resto de los combustibles. Si este incremento/decremento se mantiene en los siguientes días, la unidad de carbón en los extremos del árbol de precios tenderá a ser más costosa o más económica con respecto al resto de las unidades. Esto hará que la participación de la unidad cambie y con ello sus ganancias esperadas. Sin embargo, al no tener un tipo único de combustible en las diferentes unidades, éstas pueden verse como un portafolio, lo cual diversifica el riesgo de la compañía (Fusaro, 1998).

Las potencias de generación, así como los ingresos, costos y ganancias por periodo, para cada unidad resultan en una tabla de información arreglada como en la tabla 6 creada solamente para la unidad 2. En la misma tabla se observa que en los periodos 12 al 17, la potencia ofertada al mercado es nula, debido a que, durante dichos periodos, el precio del mercado está por debajo del costo promedio de la unidad 2; sin embargo, existe un costo asociado, lo cual es un indicativo de que la unidad no se apaga.

Para los escenarios A y B, los resultados se muestran en la tabla 7, donde la potencia no varía debido a que el precio de combustible no cambia de un periodo a otro, de manera que el costo es igual en proporción, tanto para el incremento como para el decremento del precio de electricidad.

Como se observa en la tabla 7, el escenario A presenta mayores ganancias en comparación con el escenario $\mathrm{B}$, dado que en el escenario B el precio de la electricidad cae $5 \%$.

Los ingresos, costos y ganancias totales esperadas para los escenarios A y B de un día de operación se presentan en la tabla 8, donde se observa con mayor claridad que el escenario A presenta mayores ganancias. 
Tabla 7. Ingresos, costos y ganancias esperadas de la unidad 2 escenarios A y B

\begin{tabular}{|c|c|c|c|c|c|c|c|c|c|}
\hline \multirow[b]{2}{*}{ Periodo } & \multirow[b]{2}{*}{$\begin{array}{l}\text { Potencia } \\
\text { (MW) }\end{array}$} & \multicolumn{4}{|c|}{ Escenario A } & \multicolumn{4}{|c|}{ Escenario B } \\
\hline & & $\begin{array}{l}\text { Precio } \\
\text { (\$/MWh) }\end{array}$ & $\begin{array}{c}\text { Ingresos } \\
(\$)\end{array}$ & $\begin{array}{c}\text { Costos } \\
(\$)\end{array}$ & $\begin{array}{c}\text { Ganancias } \\
(\$)\end{array}$ & $\begin{array}{l}\text { Precio } \\
(\$ / M W h)\end{array}$ & $\begin{array}{l}\text { Ingresos } \\
(\$)\end{array}$ & $\begin{array}{c}\text { Costos } \\
(\$)\end{array}$ & $\begin{array}{c}\text { Ganancias } \\
\text { (\$) }\end{array}$ \\
\hline 1 & 330 & 22.912 & 7561.08 & 4870.73 & 2690.35 & 21.58 & 7120.62 & 4870.73 & 2249.89 \\
\hline 2 & 330 & 22.897 & 7555.98 & 4774.76 & 2781.22 & 21.56 & 7115.82 & 4774.76 & 2341.06 \\
\hline 3 & 330 & 22.897 & 7555.98 & 4774.76 & 2781.22 & 21.56 & 7115.82 & 4774.76 & 2341.06 \\
\hline 4 & 330 & 22.881 & 7550.88 & 4774.76 & 2776.12 & 21.55 & 7111.02 & 4774.76 & 2336.26 \\
\hline 5 & 330 & 22.897 & 7555.98 & 4774.76 & 2781.22 & 21.56 & 7115.82 & 4774.76 & 2341.06 \\
\hline 6 & 330 & 22.912 & 7561.08 & 4774.76 & 2786.32 & 21.58 & 7120.62 & 4774.76 & 2345.86 \\
\hline 7 & 330 & 22.897 & 7555.98 & 4774.76 & 2781.22 & 21.56 & 7115.82 & 4774.76 & 2341.06 \\
\hline 8 & 310 & 20.997 & 6508.93 & 4489.45 & 2019.48 & 19.77 & 6129.77 & 4489.45 & 1640.32 \\
\hline 9 & 189 & 20.966 & 3962.51 & 2764.7 & 1197.81 & 19.74 & 3731.68 & 2764.7 & 966.98 \\
\hline 10 & 99 & 20.935 & 2072.54 & 1483.43 & 589.11 & 19.72 & 1951.81 & 1483.43 & 468.38 \\
\hline 11 & 75 & 18.818 & 1411.36 & 1141.99 & 269.37 & 17.72 & 1329.14 & 1141.99 & 187.15 \\
\hline 12 & 0 & 12.437 & 0.00 & 75.6 & -75.60 & 11.71 & 0.00 & 75.6 & -75.60 \\
\hline 13 & 0 & 13.163 & 0.00 & 75.6 & -75.60 & 12.40 & 0.00 & 75.6 & -75.60 \\
\hline 14 & 0 & 12.607 & 0.00 & 75.6 & -75.60 & 11.87 & 0.00 & 75.6 & -75.60 \\
\hline 15 & 0 & 12.870 & 0.00 & 75.6 & -75.60 & 12.12 & 0.00 & 75.6 & -75.60 \\
\hline 16 & 0 & 12.993 & 0.00 & 75.6 & -75.60 & 12.24 & 0.00 & 75.6 & -75.60 \\
\hline 17 & 0 & 12.329 & 0.00 & 75.6 & -75.60 & 11.61 & 0.00 & 75.6 & -75.60 \\
\hline 18 & 75 & 18.834 & 1412.52 & 1237.96 & 174.56 & 17.74 & 1330.23 & 1237.96 & 92.27 \\
\hline 19 & 234 & 20.966 & 4905.96 & 3405.85 & 1500.11 & 19.74 & 4620.18 & 3405.85 & 1214.33 \\
\hline 20 & 310 & 20.997 & 6508.93 & 4489.45 & 2019.48 & 19.77 & 6129.77 & 4489.45 & 1640.32 \\
\hline 21 & 330 & 22.897 & 7555.98 & 4774.76 & 2781.22 & 21.56 & 7115.82 & 4774.76 & 2341.06 \\
\hline 22 & 330 & 22.881 & 7550.88 & 4774.76 & 2776.12 & 21.55 & 7111.02 & 4774.76 & 2336.26 \\
\hline 23 & 330 & 22.897 & 7555.98 & 4774.76 & 2781.22 & 21.56 & 7115.82 & 4774.76 & 2341.06 \\
\hline 24 & 330 & 22.912 & 7561.08 & 4774.76 & 2786.32 & 21.58 & 7120.62 & 4774.76 & 2345.86 \\
\hline
\end{tabular}

Para los escenarios C y D los resultados se muestran en la tabla 9.

En la tabla 10 se observan los valores totales esperados (suma de los 24 periodos de la tabla 9). Como se ve, las ganancias en el escenario D son menores que en el C. Sin embargo, ambos escenarios son mejores que los escenarios A y B.
La figura 7 muestra en forma de árbol los cuatro escenarios para la unidad 2. El análisis se extiende para los siguientes 2 días. Como es de esperarse, al aumentar el tiempo de estudio la incertidumbre en los precios de combustible y electricidad es mayor y, con ello, las ganancias esperadas son más inciertas.

Tabla 8. Ingresos, costos y ganancias totales esperadas del escenario A y B

\begin{tabular}{cccc}
\hline Escenario & $\begin{array}{c}\text { Ingresos totales } \\
(\$)\end{array}$ & $\begin{array}{c}\text { Costos totales } \\
(\$)\end{array}$ & $\begin{array}{c}\text { Ganancias totales } \\
(\$)\end{array}$ \\
\hline $\mathrm{A}$ & 109903.59 & 72084.76 & 37818.83 \\
$\mathrm{~B}$ & 103501.4 & 72084.76 & 31416.68 \\
\hline
\end{tabular}


DOI: http://dx.doi.org/10.22201/fi.25940732e.2012.13n3.033

Estrategias operativas de compañías de generación en mercados Spot de electricidad en mercados Spot de electricidad

Tabla 9. Ingresos, costos y ganancias esperadas de la unidad 2 escenarios C y D

\begin{tabular}{|c|c|c|c|c|c|c|c|c|c|}
\hline \multirow[b]{2}{*}{ Periodo } & \multirow[b]{2}{*}{$\begin{array}{l}\text { Potencia } \\
\text { MW }\end{array}$} & \multicolumn{4}{|c|}{ Escenario C } & \multicolumn{4}{|c|}{ Escenario D } \\
\hline & & $\begin{array}{l}\text { Precio } \\
(\$ / M W h)\end{array}$ & $\begin{array}{l}\text { Ingresos } \\
(\$)\end{array}$ & $\begin{array}{c}\text { Costos } \\
(\$)\end{array}$ & $\begin{array}{c}\text { Ganancias } \\
(\$)\end{array}$ & $\begin{array}{c}\text { Precio } \\
\text { (\$/MWh) }\end{array}$ & $\begin{array}{l}\text { Ingresos } \\
(\$)\end{array}$ & $\begin{array}{c}\text { Costos } \\
(\$)\end{array}$ & $\begin{array}{c}\text { Ganancias } \\
(\$)\end{array}$ \\
\hline 1 & 330 & 22.91 & 7561.08 & 4415.99 & 3145.09 & 21.58 & 7120.62 & 4415.99 & 2704.63 \\
\hline 2 & 330 & 22.90 & 7555.98 & 4320.02 & 3235.96 & 21.56 & 7115.82 & 4320.02 & 2795.80 \\
\hline 4 & 330 & 22.88 & 7550.88 & 4320.02 & 3230.86 & 21.55 & 7111.02 & 4320.02 & 2791.00 \\
\hline 5 & 330 & 22.90 & 7555.98 & 4320.02 & 3235.96 & 21.56 & 7115.82 & 4320.02 & 2795.80 \\
\hline 6 & 330 & 22.91 & 7561.08 & 4320.02 & 3241.06 & 21.58 & 7120.62 & 4320.02 & 2800.60 \\
\hline 7 & 330 & 22.90 & 7555.98 & 4320.02 & 3235.96 & 21.56 & 7115.82 & 4320.02 & 2795.80 \\
\hline 8 & 310 & 21.00 & 6508.93 & 4061.88 & 2447.05 & 19.77 & 6129.77 & 4061.88 & 2067.89 \\
\hline 10 & 99 & 20.93 & 2072.54 & 1342.15 & 730.39 & 19.72 & 1951.81 & 1342.15 & 609.66 \\
\hline 11 & 75 & 18.82 & 1411.36 & 1033.22 & 378.14 & 17.72 & 1329.14 & 1033.22 & 295.92 \\
\hline 12 & 0 & 12.44 & 0.00 & 68.4 & -68.40 & 11.71 & 0.00 & 68.4 & 68.40 \\
\hline 13 & 0 & 13.16 & 0.00 & 68.4 & -68.40 & 12.40 & 0.00 & 68.4 & 68.40 \\
\hline 14 & 0 & 12.61 & 0.00 & 68.4 & -68.40 & 11.87 & 0.00 & 68.4 & 68.40 \\
\hline 15 & 0 & 12.87 & 0.00 & 68.4 & -68.40 & 12.12 & 0.00 & 68.4 & 68.40 \\
\hline 16 & 0 & 12.99 & 0.00 & 68.4 & -68.40 & 12.24 & 0.00 & 68.4 & 68.40 \\
\hline 17 & 0 & 12.33 & 0.00 & 68.4 & -68.40 & 11.61 & 0.00 & 68.4 & 68.40 \\
\hline 18 & 75 & 18.83 & 1412.52 & 1129.19 & 283.33 & 17.74 & 1330.23 & 1129.19 & 201.04 \\
\hline 19 & 234 & 20.97 & 4905.96 & 3081.48 & 1824.48 & 19.74 & 4620.18 & 3081.48 & 1538.70 \\
\hline 21 & 330 & 22.90 & 7555.98 & 4320.02 & 3235.96 & 21.56 & 7115.82 & 4320.02 & 2795.80 \\
\hline 22 & 330 & 22.88 & 7550.88 & 4320.02 & 3230.86 & 21.55 & 7111.02 & 4320.02 & 2791.00 \\
\hline 23 & 330 & 22.90 & 7555.98 & 4320.02 & 3235.96 & 21.56 & 7115.82 & 4320.02 & 2795.80 \\
\hline 24 & 330 & 22.91 & 7561.08 & 4320.02 & 3241.06 & 21.58 & 7120.62 & 4320.02 & 2800.60 \\
\hline
\end{tabular}

Tabla 10. Ingresos, costos y ganancias totales esperadas del escenario C y D

\begin{tabular}{cccc}
\hline & $\begin{array}{c}\text { Ingresos totales } \\
(\$)\end{array}$ & $\begin{array}{c}\text { Costos totales } \\
(\$)\end{array}$ & $\begin{array}{c}\text { Ganancias totales } \\
(\$)\end{array}$ \\
\hline $\mathrm{C}$ & 109903.6 & 65237.79 & 44665.8 \\
$\mathrm{D}$ & 103501.44 & 65237.79 & 38263.65 \\
\hline
\end{tabular}




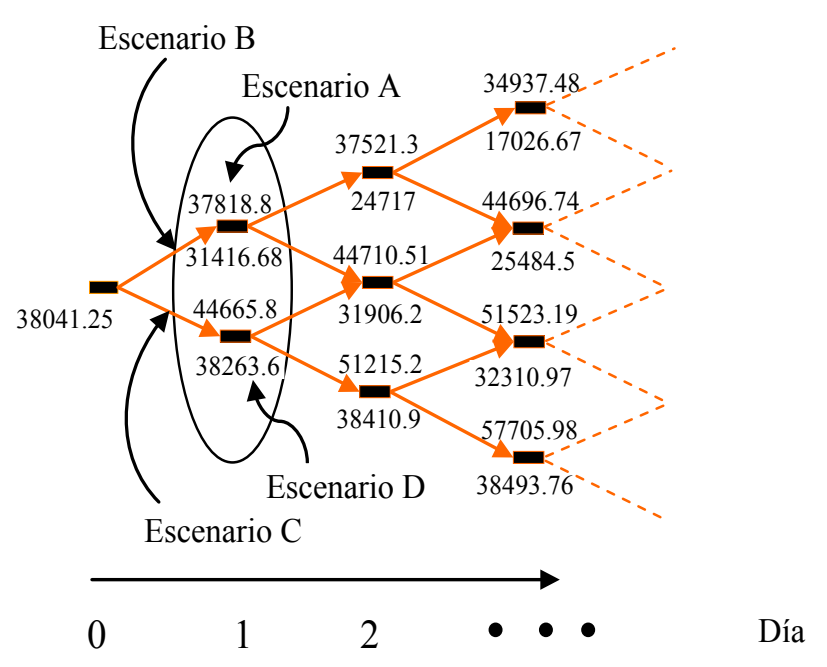

Figura7. Árbol de beneficios para los escenarios A, B, C y D

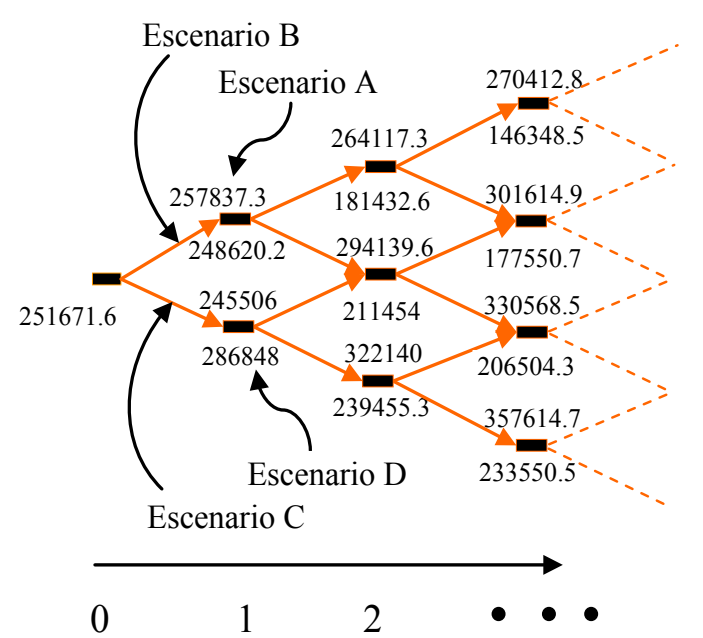

Día

Figura 8. Árbol de beneficios para los escenarios A, B, C y D de la GenCo

La figura 8 muestra los beneficios para la GenCo en los cuatro escenarios. Estas ganancias son el resultado de la suma de las ganancias individuales de las unidades a lo largo de los 24 periodos. Se puede apreciar que se llegará a tener pérdidas en el caso $\mathrm{B}$, donde el precio del combustible sube demasiado y, al mismo tiempo, disminuye el precio de la electricidad.

\section{Conclusiones}

En un sector eléctrico tradicional (modelo verticalmente integrado), el precio de la electricidad está directamente relacionado con el precio de los combustibles. En un ambiente de mercado, existe una componente adi- cional que es la estrategia de cada compañía de generación, es decir, aun cuando el precio de los combustibles sea información pública, las variaciones en el precio puede o no indexarse tal cual en los costos de producción de las unidades de los competidores.

Las variaciones en los precios de los combustibles están correlacionadas con el precio de electricidad. Una correlación positiva entre ambos puede hacer que las ganancias esperadas se reduzcan si el precio de la electricidad se incrementa en menor proporción que el del combustible, por lo que se tendrá un mayor riesgo de obtener los retornos esperados. Por otro lado, cuando el incremento en el precio de electricidad es mayor que el incremento en el precio de combustible, las ganancias esperadas se incrementan. Un efecto similar, pero inverso, se presenta cuando la correlación entre el precio de electricidad y de combustible es negativa.

\section{Agradecimientos}

Eliseo Valmiki Liera-Moreno agradece al Consejo Nacional de Ciencia y Tecnología CONACyT, por el apoyo económico para la realización de los estudios de maestría. Asimismo, agradece a la Dirección General de Educación Superior Tecnológica (DGEST) por el apoyo en la terminación de los mismos, como becario con registro No. 052007093-FOG.

\section{Referencias}

Bhattacharya K., Bollen M.H.J., Daalder J.E. Operation of Restructured Power Systems, Kluwer Academic Publishers, 2001.

Blaesing B., Hartmann Th., Haubrich H.J. (2007), Valuation of Generation Assets using Risk Management Methods, IEEE Power Tech, 2007.

Chung-Li T.S. Exercising Real Unit Operational Options under Price Uncertainty. 2000 IEEE Power Engineering Society Winter Meeting, volumen 1, 2000: 436-440.

Dimitris N. Reliability Constrained Unit Commitment Using Simulated Annealing. IEEE Trans. On Power System, volumen 21 (número 4), noviembre de 2006: 1699-1706.

Fusaro P.C. Energy Risk Management: Hedging Strategies and Instruments for the International Energy Markets, McGraw-Hill, 1998.

GAMS (2004). The Solver Manuals.

Gutierrez-Alcaraz G. Sequential Time-Step Generation Companies Decisions in Oligopolistic Electricity Market. Electric Power Systems Research, volumen 78 (número 5), mayo de 2008: 824-834.

Gwo-Ching L., Ta-Peng T. A Novel GA-Based and Meta-Heuristics Method for Short-Term Unit Commitment Problem, en: 2004 IEEE Power Engineering Society General Meeting, volumen 1, 2004, pp. 1088-1093. 
Hobbs B.F., Rothkopf M.H., O'Neill R.P., Chao H.P. The Next Generation of Electric Power Unit Commitment Models, KIuwer Academic Publishers, 2001.

Hossein H., Hossein S., Ashkan R.K. On the Self-Scheduling of a Power Producer in Uncertain Trading Environments. Electric Power System Research, volumen 78 (número 3), marzo de 2008: 311-317.

Joskow P.L. Regulation and Deregulation after 25 Years: Lessons Learned for Research in Industrial Organization. Review of Industrial Organization, volumen 26 (número 2), marzo de 2005: 169-193.

Padhy N.P. Unit Commitment Problem under Deregulated Environment-A Review. IEEE Trans. On Power System, volumen 19, (número 2), mayo de 2004: 1196-1205.

Paiva-Mendes D. Generation Scheduling, Pricing Mechanisms and Bidding Strategies in Competitive Electricity Markets, tesis (Ph. D. dissertation), University of Manchester, Institute of Science and Technology, 1999.

Sheblé G.B., Fahd G.W. Unit Commitment Literature Synopsis. IEEE Trans. On Power System, volumen 9 (número 1), 1994: 128-135.

Sheblé G.B. Computacional Auction Mechanisms for Restructure Power Industry Operationa (Power Electronics and Power Systems, springer, 1999.

Vijay K. Auction Theory, 1st ed., Academic Press, 2002.

Wen F.S., David A.K. Strategic Bidding for Electricity Supply in Day-Ahead Energy Market. Electric Power System Research, volumen 59, 2001: 197-206.

Wilson R. Architecture of Power Markets, Research Paper Núm. 1708, Graduate School of Business, Stanford University, 2001.
Wood A., Wollenberg B. Power Generation, Operation and Control, 2a ed., John Wiley \& Sons, 1996.

Zou B., Maosong Y., Xie X. The Comparisons between Pricing Methods on Pool-Based Electricity Market Using Agent-Based Simulation, en: Proceedings of the 2004 IEEE International Conference on Electric Utility Deregulation, Restructuring and Power Technologies, abril de 2004, (DRPT 2004), pp. 285-289.

Zuyi L. Security-Constrained Unit Commitment for Simultaneous Clearing of Energy and Ancillary Services Markets. IEEE Trans. On Power System, volumen 20 (número 2), mayo de 2005: 1079-1088.

\section{Este artículo se cita:}

\section{Citación Chicago}

Liera-Moreno, Eliseo Valmiki, Guillermo Gutiérrez-Alcaraz, José Horacio Tovar-Hernández. Estrategias operativas de compañías de generación en mercados Spot de electricidad. Ingeniería Investigación y Tecnología, XIII, 03, (2012): 351-364.

\section{Citación ISO 690}

Liera-Moreno E.V., Gutiérrez-Alcaraz G., Tovar-Hernández J.H. Estrategias operativas de compañías de generación en mercados Spot de electricidad. Ingeniería Investigación y Tecnología, volumen XIII (número 3), julio-septiembre 2012: 351-364.

\section{Semblanza de los autores}

Eliseo Valmiki-Liera. Realizó los estudios de licenciatura y maestría en el Instituto Tecnológico de Morelia (ITM), obteniendo los grados respectivos en 2006 y 2007. Actualmente labora como jefe de operación del Departamento de Distribución en la División Valle de México Norte en la zona Cuautitlán de la Comisión Federal de Electricidad.

Guillermo Gutiérrez-Alcaraz. Realizó los estudios de licenciatura y maestría en el Instituto Tecnológico de Morelia. Obtuvo el doctorado en ingeniería eléctrica por la Universidad Estatal de Iowa. Actualmente es profesor del PGIIE del ITM.

José Horacio Tovar-Hernández. Realizó los estudios de licenciatura en el Instituto Tecnológico de Morelia (ITM), obteniendo el grado de ingeniero electricista en 1984. Obtuvo la maestría y el doctorado en ingeniería eléctrica en la Sección de Estudios de Posgrado e Investigación de la Escuela Superior de Ingeniería Mecánica y Eléctrica del Instituto Politécnico Nacional, obteniendo los grados respectivos en 1989 y en 1995. Actualmente es profesor del programa de graduados e investigación en ingeniería eléctrica (PGIIE) del ITM. 